\title{
Nontargeted nuclear magnetic resonance (NMR) analysis to detect hazardous substances including methanol in unrecorded alcohol from Novosibirsk, Russia
}

\author{
Thomas Hausler ${ }^{\mathrm{a}, \mathrm{b}}$, Alex O. Okaru, ${ }^{\mathrm{a}, \mathrm{c}}$, Maria Neufeld ${ }^{\mathrm{de}, \mathrm{e}}$, Jürgen Rehm ${ }^{\mathrm{d}, \mathrm{e}}$, Thomas Kuballa $a^{\mathrm{a}}$, Burkhard Luy ${ }^{\mathrm{b}}$ and Dirk W. Lachenmeier, ${ }^{\mathrm{a}, \mathrm{e},}$ \\ ${ }^{a}$ Chemisches und Veterinäruntersuchungsamt (CVUA) Karlsruhe, Weissenburger Straße 3, 76187 Karlsruhe, Germany. \\ E-mails: thomas.hausler@web.de; thomas.kuballa@cvuaka.bwl.de; Corresponding Author: lachenmeier@web.de \\ ${ }^{b}$ Institute of Organic Chemistry and Institute for Biological Interfaces 4 - Magnetic Resonance, Karlsruhe Institute of Technology (KIT), \\ Fritz-Haber-Weg 6, 76131 Karlsruhe, Germany. E-mail: burkhard.luy@kit.de \\ 'Department of Pharmaceutical Chemistry, University of Nairobi, Off Ngong Road, Nairobi, P.O. Box 19676-00202 Nairobi, Kenya. \\ E-mail: aokaru@uonbi.ac.ke
}

${ }^{\mathrm{d} C e n t r e}$ for Addiction and Mental Health (CAMH), 33 Russell Street, Toronto, ON M5S 2S1 Canada: E-mails: neufeld.maria@googlemail.com; jtrehm@gmail.com

${ }^{\mathrm{e}}$ Institute for Clinical Psychology and Psychotherapy, TU Dresden, Chemnitzer Str. 46, 01187 Dresden, Germany.

\begin{abstract}
Nuclear magnetic resonance (NMR) spectroscopy was applied to the analysis of alcoholic products in the context of health and safety control. A total of 86 samples of unrecorded alcohol were collected in Novosibirsk and nearby cities in Russia. Sampling was based on interviews with alcohol dependent patients, and unrecorded alcohol thus defined included illegally or informally produced alcoholic products (e.g., counterfeit or home-made alcoholic beverages) or surrogate alcohol in the form of cosmetic or medicinal products and industrial non-beverage alcohol such as antifreeze (antifreeze windshield washer fluid). For sample preparation, addition of buffer and of a water/ethanol mixture was required as single step. To detect potentially harmful samples, a nontargeted approach based on principal component analysis (PCA) was applied. The PCA scores plot shows six conspicuous samples with highly divergent scores from the rest. These samples are antifreeze windshield washer fluids containing high amounts of methanol, with concentrations in a range between $7 \%$ and $48 \%$ vol. The antifreeze products were bought in regular retail sale and were claimed as "not containing methanol" on five out of six labels. Additionally, formic acid (1.1\%) was observed in four of the alcohol-containing medicinal products. The major advantage of NMR over conventional methods is the fact that it not only provides the same quantitative data for specific compounds, but also allows rapid nontargeted screening for unknown contaminants.
\end{abstract}

\section{Introduction}

he increasing use of nuclear magnetic resonance (NMR) spectroscopy for the analysis of beverages is attributed to the ability of the technique to selectively determine individual components in complex mixtures and providing much richer chemical information than conventional analyses ${ }^{1,2}$. The technique has gained popularity in the screening of beverages for components for authentication purposes ${ }^{3}$, as well as routine analysis and rapid detection of potentially unsafe substances ${ }^{4-7}$. As a result of the minimal sample preparation and the short analysis time, the sample output is considerably increased compared to other approaches ${ }^{1,8-10}$.

The consumption of surrogate alcohol, a subgroup of unrecorded alcohol, continues to endanger public health in Russia ${ }^{11}$, mainly because of high ethanol content and lower prices compared to recorded alcohol. Surrogate alcohol is officially not intended for human consumption, but is consumed as a surrogate for alcoholic beverages, often by people with a low socioeconomic status and alcohol dependence ${ }^{11-14}$. The World Health Organisation (WHO) estimated that out of the $15.1 \mathrm{~L}$ total adult per capita consumption of pure alcohol in the Russian Federation, 3.6L were unrecorded $(24 \%)^{15}$. Other estimates were considerably higher ${ }^{11}$.
Previous studies conducted on surrogate alcohol from Russia detected high levels of disinfectants and denaturing agents in some samples, which pose an additional risk beyond ethanol ${ }^{16,17}$. The aim of this research was to use principal component analysis (PCA) of NMR spectral data to rapidly detect potentially dangerous and harmful substances in surrogate alcohol samples obtained from Novosibirsk and surroundings.

\section{Materials and methods}

A total of 86 samples consisting of different types of unrecorded alcohols were collected from typical sale locations of unrecorded alcohol, such as kiosks, taxi cabs, pharmacies or private homes in Russia in the context of an international project designed to explore drinking patterns and quality of unrecorded alcohol and their consequences for the consumer's health. The samples included homemade beverages, food flavouring agents based on ethanol, smuggled alcohol, alleged counterfeits, obvious counterfeits, medicinal and cosmetic products containing alcohol as well as diluted nonbeverage alcohol such as antifreeze washer fluids, which may also be drunk by people of low socioeconomic status ${ }^{18}$.

Samples for NMR spectroscopy were prepared by diluting $300 \mu \mathrm{l}$ of the beverage with $240 \mu \mathrm{l}$ of a water/ethanol mixture (190/50) 
and $60 \mu \mathrm{l}$ of $\mathrm{NMR}$ buffer $\left(\mathrm{pH} 7.45,1.5 \mathrm{M} \mathrm{KH}_{2} \mathrm{PO}_{4}\right.$ in $\mathrm{D}_{2} \mathrm{O}, 0.1 \%$ 3-(trimethylsilyl)-propionate acid-d4 (TSP), $3 \mathrm{mM} \mathrm{NaN}_{3}$ ) before measurement on a Bruker Avance 400 Ultrashield spectrometer (Bruker Biospin, Rheinstetten, Germany) equipped with a 5-mm SEl probe with Z-gradient coils using a Bruker Automatic Sample Changer (Sample Xpress). The spectra were automatically acquired at 300.0 K under the control of ICON-NMR (Bruker BioSpin, Rheinstetten, Germany), requiring about 25 min per sample. A suppression of eight frequencies (around $4.70 \mathrm{ppm}$ for $\mathrm{OH}$-group signal for both water and ethanol, $3.66 \mathrm{ppm}$ for ethanol $\mathrm{CH}_{2}$ quartet, and $1.18 \mathrm{ppm}$ for the corresponding ethanol $\mathrm{CH}_{3}$ triplet) was conducted. The measurement protocol was previously described in detail ${ }^{8,9}$. All ${ }^{1} \mathrm{H}-\mathrm{NMR}$ spectra were automatically phase and baseline corrected using the Topspin 3.2 software package (Bruker BioSpin, Rheinstetten, Germany).

Principal component analysis (PCA) was performed using the Unscrambler $X$ software (CAMO Software AS, Oslo, Norway). Bucketing of spectra data was done with Amix 3.9.14 (Bruker BioSpin, Rheinstetten, Germany) with a 0.01 ppm width as previously described for alcoholic beverages ${ }^{3}$. The chemical shift regions of -0.5 to $0.3,1.0$ to $1.39,3.43$ to 3.87 and 4.78 to 5.07 ppm containing TSP, ethanol and the water signals, respectively, were excluded. Validation of the PCA model was done by full cross validation with 20 segments, each segment containing three samples.

\section{Results and discussion}

\section{Nontargeted and targeted analysis of unrecorded alcohol samples}

The scores plot of the PCA with all samples shows a clear grouping of six outliers (Figure 1). The highest PC1 values on the scores plot are attributable to six antifreeze products. Examination of the loadings plots (Figure 2) and corresponding NMR spectra shows that only the methanol signal at $3.36 \mathrm{ppm}$ has significant influence on the PC1 score. After the methanol-containing antifreeze samples are eliminated as outliers from the PCA analysis, the scatter plot of the remaining unrecorded alcohols gives another definite cluster of divergent samples (Figure 3). As depicted by the loadings plot of PC2 (Figure 4), the score of PC2 is primarily negatively determined by two NMR signals at $8.15 \mathrm{ppm}$ (ethyl formate) and $8.48 \mathrm{ppm}$ (formic acid). All four medicinal alcohols, containing formic acid as labeled, are grouped with a high PC2 value. The loadings plot of PC2 indicates that the value of PC2 is determined by the amount of formic acid in the samples.

Following the non-targeted PCA analysis, quantification of the samples for the identified compounds methanol, formic acid and ethyl formate was conducted by use of the internal standard TSP using the signals at 3.36, 8.15 and $8.48 \mathrm{ppm}$ for methanol, formic acid and ethyl formate, respectively. The quantitative results are separately discussed in the following sections.

\section{Methanol in antifreeze samples}

The antifreeze compounds were found to contain $7 \%$ vol, $26 \%$ vol, $26 \%$ vol, $27 \%$ vol, $28 \%$ vol, and $48 \%$ vol methanol. The content of methanol in these antifreeze samples was alarmingly high and exceeded the maximum tolerable concentration (MTC) of methanol of $2 \%$ vol in all cases ${ }^{19}$. There was no label information about methanol as an ingredient in any of the six products, and five of them claimed to "not containing methanol" on the labels. This poses a particular risk to the consumer, who may be exposed to such products accidentally because of lack of information on the label or because of incorrect and misleading labelling of the products. The incorrect and deceptive labelling is perhaps to avoid scrutiny by the Russian authorities since the use of methanol in antifreeze has been banned since 2000 in Russia ${ }^{18}$. Such alcohol products continue to pose considerable threat to the health of consumers of surrogate alcohol, a majority of whom belong to low socio-economic strata, including but not limited to marginalized groups such as people with alcohol dependence, who may be attracted to these products because of their low cost ${ }^{11,18}$. This is corroborated by a recent study highlighting the widespread consumption of cheap unrecorded

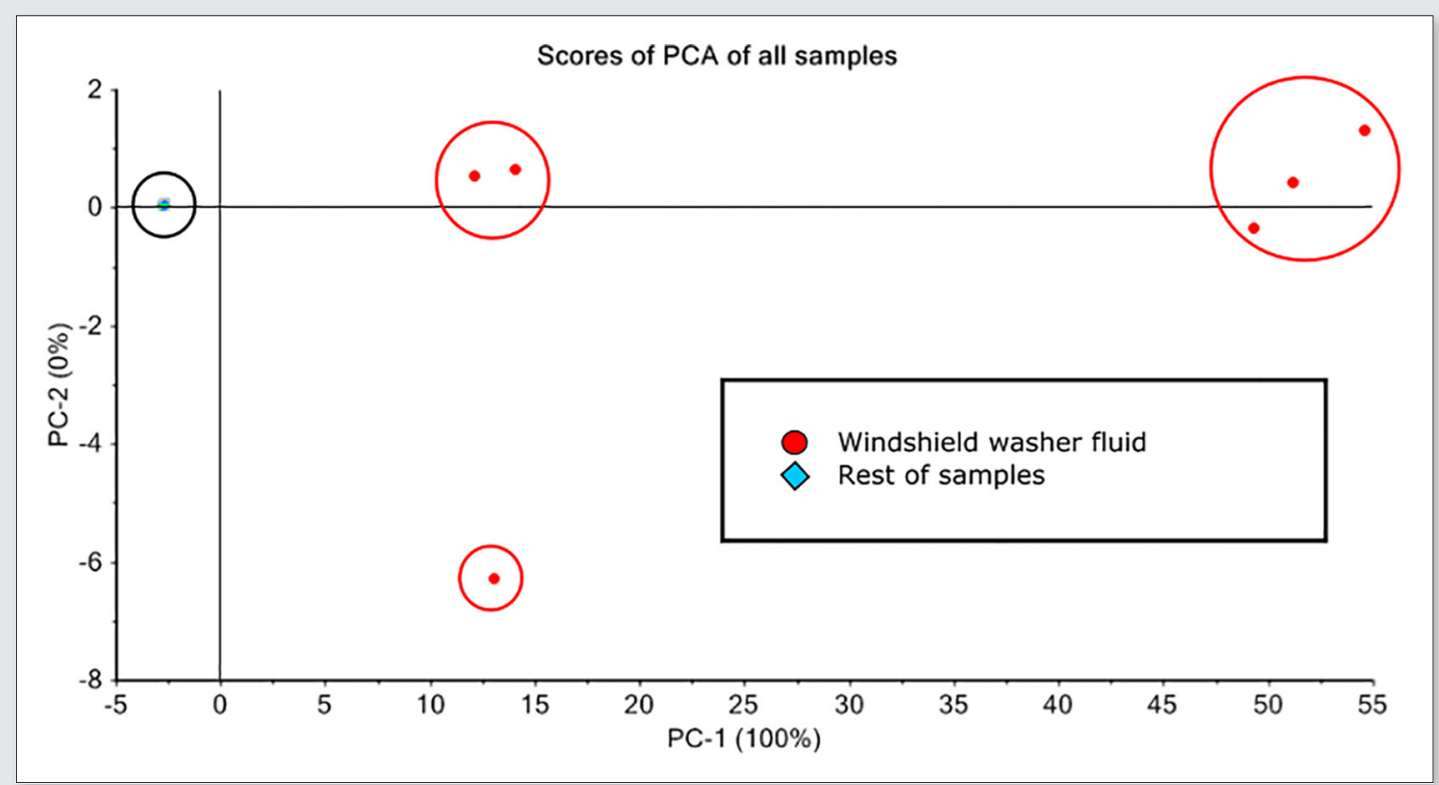

Figure 1. PCA scores plot of all measured samples. All unrecorded alcohols containing methanol (red circles) show a different PC-1 value compared to the rest of the samples (black circle, including vodka, homemade alcohol, cosmetic alcohol, medicinal alcohol, beer, food flavouring agent, counterfeited alcohol). (Note: the scores of the rest of the samples in the green circle are so similar that individual samples cannot be distinguished in this visualization). 


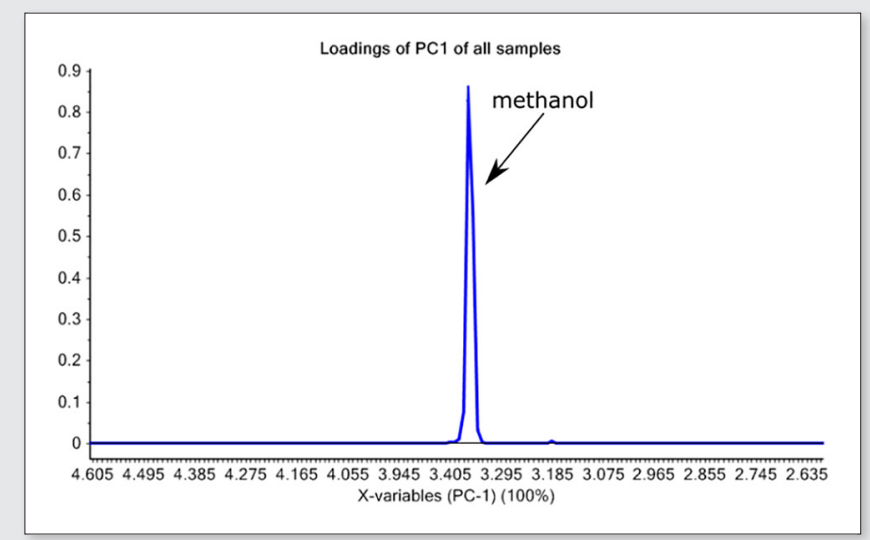

Figure 2. Loadings plot of PC-1 of all measured samples. The value of PC-1 is only determined by the amount of methanol (NMR-signal at $3.36 \mathrm{ppm}$ and ${ }^{13} \mathrm{C}$ coupling signal at $\left.3.18 \mathrm{ppm}\right)$.

beverages such as medicinal or cosmetic alcoholic tinctures by marginalized groups ${ }^{18}$.

\section{Formic acid in medicinal surrogates}

The amounts of formic acid and ethyl formate were detected at $1.1 \mathrm{~g} / 100 \mathrm{~g}$ and $0.5 \mathrm{~g} / 100 \mathrm{~g}$, respectively. The four samples, containing formic acid, are medicinal tinctures called "Formic alcohol" and are meant for medicinal purposes (e.g. as local antiseptic and claimed for treatment of myositis, neuralgia and specific types of arthritis). However, due to its cheap price and relatively large vessel size, formic alcohol is also frequently consumed as surrogate alcohol by alcohol dependent individuals. While methanol has been a common element occasionally occurring in unrecorded alcohol and leading to a number of documented poisoning cases (see review in Lachenmeier et al. ${ }^{20}$ ), formic acid so far has never before been appreciated as potential constituent of unrecorded alcohol. Formic acid is an oxidation product of methanol, while ethyl formate is a

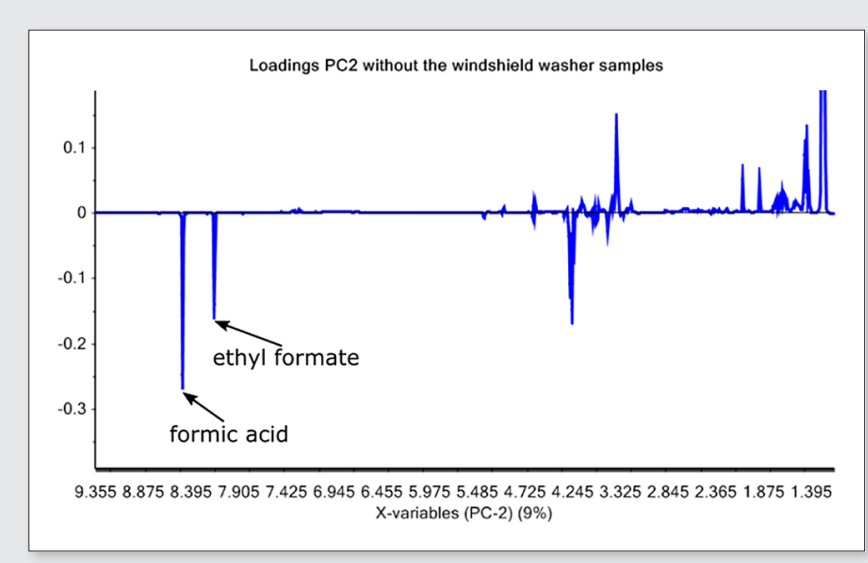

Figure 4. Loadings plot of all samples without the windshield fluid samples. The plot indicates, that the value of PC2 is mainly negatively determined by the amount of formic acid (NMR signal at $8.48 \mathrm{ppm}$ ) and of ethyl formate (NMR signal at $8.15 \mathrm{ppm}$ ). PC1 (not shown here) is determined mainly by the content of glucose in the different samples.

flavor compound of rum and raspberry ${ }^{21}$ that arises from the esterification of formic acid in excess ethanol, a reaction favored by the high content of ethanol in the medicinal products. The use of formic acid (E 236) as food additive has been banned in the European Union (EU) since 1998. Formic acid is also not currently approved in any medicinal product in the EU, while it has been formerly applied in hair tonics to stimulate hair growth (5-10\% formic acid solution), especially in alopecia areata ${ }^{22}$. The Joint FAO/WHO Expert Committee on Food Additives (JECFA) has suggested a group acceptable daily intake (ADI) for formic acid and ethyl formate of $3 \mathrm{mg} / \mathrm{kg}$ bodyweight per day ${ }^{23}$. For a $60 \mathrm{~kg}$ person, this would be $180 \mathrm{mg}$ per day. For the surrogate alcohol containing $1.4 \mathrm{~g} / 100 \mathrm{~g}$ (sum of formic acid and ethyl formate, expressed as formic acid), approximately $13 \mathrm{~g}$ (about $1 / 4$ of a bottle) need to be consumed to exceed the ADI. It should be noted that the JECFA ADI is conservatively estimated using safety factors of 100 , so that acute toxic effects

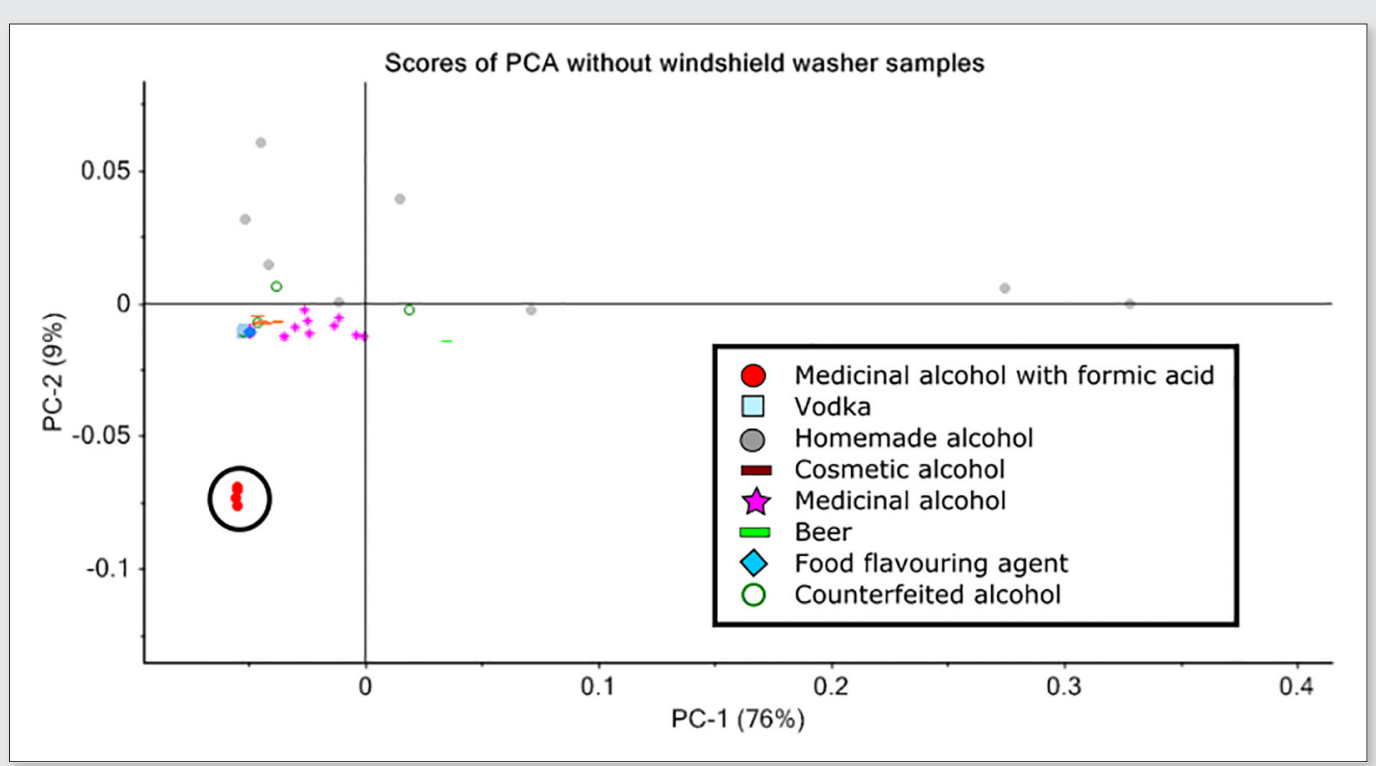

Figure 3. Scores plot of all samples without the six windshield fluid samples. All four samples, containing formic acid and ethyl formate as labelled, shows the same behaviour in their PC-2 value. 
are not expected even at this level of intake. Serious formic acid poisoning (reversible intravascular coagulation and acute renal failure) was only observed following intake of $30 \mathrm{~g}$ of formic acid or more, and high mortality (14 out of 15 patients) was recorded for ingestion of 45 to $200 \mathrm{~g}$ of formic acid $^{24}$. These levels of intake cannot be reached with the alcohol surrogates. Chronic effects may be the exacerbation of the effects of ethanol contributing to metabolic acidosis, which appears to be a common denominator for consumers of surrogate alcohol. For example, we have previously determined that consumption of mouthwash may contribute to metabolic acidosis due to methyl salicylate contents ${ }^{25}$. For this reason, we believe that the benefits of medicinal products containing formic acid (and ethyl formate) do not outweigh their risks. Similar to the prohibition of unsafe compounds such as diethyl phthalate (DEP) and polyhexamethyleneguanidine (PHMG) in alcohol-containing disinfectants due to the risk of human ingestion ${ }^{26}$, we suggest that regulators in Russia should reassess the safety and efficacy of medicinal products containing formic acid and potentially phaseout their marketing authorisation or at least reduce the approved bottle sizes or increase their price.

\section{Conclusion}

NMR-spectroscopy was found to be a suitable technique for rapid nontargeted analysis of a large number of spirit samples. A similar study conducted in 2010 on surrogate alcohol samples from Russia utilizing multivariate data analysis detected two samples out of 22 to contain the toxic denaturant diethyl phthalate (DEP) and the disinfectant polyhexamethyleneguanidine $(\mathrm{PHMG})^{16}$. In contrast, in this study methanol in windshield fluid samples and formic acid in medicinal surrogates are detected as hazardous substances. However, none of the current 86 samples contained DEP and PHMG. As all measured antifreeze samples contained considerable amounts of methanol, which is a banned substance for antifreeze in Russia, quality control of corresponding products is strongly advised. The current findings reveal that surrogate alcohol continues to pose significant hazards to the lives of problem drinkers and alcohol dependent people from low socio-economic strata in Russia.

With the possibility of simultaneous analysis of a large number of samples, the PCA approach is highly effective in the field of industrial analysis and quality control. The combination of NMR and PCA for data analysis is therefore a fast and easy-to-implement method for the investigation of a large number of unknown components within a certain concentration in alcohol samples without a priori knowledge of their chemical structure. Although infrared spectroscopy as an alternative may be less time consuming, NMR spectra show less overlap of signals and achieve improved identification based on specific chemical shifts and coupling patterns. Due to the minimal sample preparation effort and the possibility of multiple solvent suppression of ethanol and water signals, NMR spectroscopy is the method of choice in the field of nontargeted analysis of potentially hazardous substances in alcoholic beverages. The technique will be hopefully applied by governmental and industrial quality control institutions in the future to increase consumer protection.

\section{References}

1. M. Lees and B. Popping, Food Authenticity and Traceability. CRC Press LLC, Wahington DC, USA. pp. 347-356 (2003). doi: http://dx. doi. org/10.1533/9781855737181.2.347
2. C. Simmler, J.G. Napolitano, J.B. McAlpine, S.N. Chen and G.F. Pauli, "Universal quantitative NMR analysis of complex natural samples", Curr. Opin. Biotechnol. 25, 51-59 (2014). doi: http://dx.doi.org/10.1016/j. copbio.2013.08.004

3. D.W. Lachenmeier, W. Frank, E. Humpfer, H. Schäfer S. Keller, M. Mortter and M. Spraul, "Quality control of beer using high-resolution nuclear magnetic resonance spectroscopy and multivariate analysis", Eur. Food Res. Technol. 220, 215-221 (2005). doi: http://dx.doi.org/10.1007/ s00217-004-1070-7

4. Y.B. Monakhova, B. Schütz, H. Schäfer, M. Spraul M, T. Kuballa, H. Hahn and D.W. Lachenmeier, "Validation studies for multicomponent quantitative NMR analysis: the example of apple fruit juice", Accredit. Qual. Assur. 19, 17-29 (2013). doi: http://dx.doi.org/10.1007/s00769013-1026-3

5. Y.B. Monakhova, T.Kuballa and D.W. Lachenmeier, "Rapid quantification of ethyl carbamate in spirits using NMR spectroscopy and chemometrics", ISRN Anal. Chem. 2012, Article ID 989174 (2012). doi: http:// dx.doi.org/10.5402/2012/989174

6. K. Wegert, Y.B. Monakhova, T. Kuballa, H. Reusch, G. Winkler and D.W. Lachenmeier, "Regulatory control of energy drinks using $1 \mathrm{H}$ NMR spectroscopy", Lebensmittelchem. 66, 143-145 (2012). doi: http:// dx.doi.org/10.1002/lemi.201290113

7. M. Wunsch, T. Kuballa and H. Reusch, " $1 \mathrm{H}$ NMR screening of bubble tea . Free from ' poisonous traces ', nevertheless full of undeclared additives", Deut. Lebensm. Rundsch. 109, 427-431 (2015).

8. Y.B. Monakhova, T. Kuballa and D.W. Lachenmeier, "Nontargeted NMR analysis to rapidly detect hazardous substances in alcoholic beverages", Appl. Magn. Reson. 42, 343-352 (2012). doi: http://dx.doi.org/10.1007/ s00723-011-0309-2

9. Y.B. Monakhova, H. Schäfer, E. Humpfer, M. Spraul, T. Kuballa and D.W. Lachenmeier, "Application of automated eightfold suppression of water and ethanol signals in $1 \mathrm{H}$ NMR to provide sensitivity for analyzing alcoholic beverages", Magn. Reson. Chem. 49, 734-739 (2011). doi: http://dx.doi.org/10.1002/mrc.2823

10. Y.B. Monakhova, T. Kuballa and D.W. Lachenmeier, "Chemometric methods in NMR spectroscopic analysis of food products", J. Anal. Chem. 68, 755-766 (2013). doi: http://dx.doi.org/10.1134/S1061934813090098

11. J. Rehm, K. Shalini, E. Larsen, M.X. Rehm, A.V. Samokhvalov, K.D. Shield, M. Roerecke and D.W. Lachenmeier, "A systematic review of the epidemiology of unrecorded alcohol consumption and the chemical composition of unrecorded alcohol", Addiction 109, 880-893 (2014). doi: http://dx.doi.org/10.1111/add.12498

12. D.W. Lachenmeier, J. Lietz, K. Schoeberl, T. Kuballa, I. Straub and J. Rehm, "Quality of illegally and informally produced alcohol in Europe: Results from the AMPHORA project", Adicciones 23, 133-140 (2011). http://dx.doi.org/10.20882/adicciones.156

13. P. Anderson, L. Møller and G. Galea, Alcohol in the European Union: Consumption, Harm and Policy Issues", WHO Regional Office for Europe, Copenhagen, Denmark. pp. 1-142 (2012)

14. V. Radaev, Impact of a new alcohol policy on homemade alcohol consumption and sales in Russia", Alcohol Alcohol. 50, 365-372. (2014). doi: http://dx.doi.org/10.1093/alcalc/agv008

15. WHO. "Global Status Report on Alcohol and Health," World Health Organization, Geneva, Switzerland. pp. 290. (2014)

16. Y.B. Monakhova, T. Kuballa, J. Leitz and D.W. Lachenmeier, "Determination of diethyl phthalate and polyhexamethylene guanidine in surrogate alcohol from Russia", Int. J. Anal. Chem. 2011, Article ID 704795 (2011). doi: http://dx.doi.org/10.1155/2011/704795 
17. D.W. Lachenmeier, Y.B. Monakhova, A.V. Samokhvalov and J. Rehm, "Causality between polyhexamethyleneguanidine occurrence in unrecorded alcohol and cholestatic hepatitis outbreak in Russia", Clin. Toxicol. 50, 154-155 (2012). doi: http://dx.doi.org/10.3109/15563650. 2011.646355

18. M. Neufeld, D.W. Lachenmeier, T. Hausler and J. Rehm, "Surrogate alcohol containing methanol, social deprivation and public health in Novosibirsk, Russia", Int. J. Drug Policy 2016. doi: http://dx.doi. org/10.1016/j.drugpo.2016.08.001

19. A.J Paine and A.D. Dayan, "Defining a tolerable concentration of methanol in alcoholic drinks," Hum. Exp. Toxicol. 20, 563-568. (2001). doi: http://dx.doi.org/10.1191/096032701718620864

20. D.W. Lachenmeier, J. Rehm and G. Gmel, "Surrogate alcohol: what do we know and where do we go?", Alcohol Clin. Exp. Res. 31, 1613-24 (2007). doi: http://dx.doi.org/10.1111/j.1530-0277.2007.00474.x

21. International Programme on Chemical Safety. World Health Organization. Safety evaluation of certain food additives and contaminants. WHO Food Additives Series 40, "Saturated aliphatic acyclic linear primary alcohols, aldehydes and acids", Prepared by: The forty-ninth meeting of the Joint FAO/WHO Expert Committee on Food Additives (JECFA). World Health Organization, Geneva (1998). http://www.inchem.org/ documents/jecfa/jecmono/v040je10.htm

22. A. Perutz, C. Siebert and R. Winternitz., Pharmakologie der Haut. Arzneimittel. Allgemeine Therapie. Julius Springer, Berlin, Germany (1930).

23. H. Panda, Perfumes and flavours technology handbook. Asia Pacific Business Press Inc. Delhi, India (2010).

24. D.B. Jefferys and H.M. Wiseman, "Formic acid poisoning", Postgrad. Med. J. 56, 761-762 (1980). doi: http://dx.doi.org/10.1136/ pgmi.56.661.761

25. D.W. Lachenmeier, Y.B. Monakhova, M. Markova, T. Kuballa and J. Rehm, "What happens if people start drinking mouthwash as surrogate alcohol? A quantitative risk assessment," Food Chem. Toxicol. 51, 173178 (2013). doi: http://dx.doi.org/10.1016/i.fct.2012.09.031

26. Y.V. Solodun, Y.B. Monakhova, T. Kuballa, A. Samokhvalov, J. Rehm and D.W. Lachenmeier, "Unrecorded alcohol consumption in Russia: toxic denaturants and disinfectants pose additional risks", Interdiscip. Toxicol. 4, 198-205 (2011). doi: http://dx.doi.org/10.2478/v10102-011-0030-x 